\title{
Temporal lobe factor in verb fluency Raffaella Öckinger*
}

\author{
Address: Neurotec Institution, Karolinska Institute, Sweden \\ * Corresponding author
}

\author{
from International Society on Brain and Behaviour: 2nd International Congress on Brain and Behaviour \\ Thessaloniki, Greece. 17-20 November 2005 \\ Published: 28 February 2006 \\ Annals of General Psychiatry 2006, 5(SuppI I):SI II doi:I0.I I86/I744-859X-5-SI-SI I I
}

\section{Background}

This study evaluated whether decreased word fluency in Mild Cognitive Impairment and related disorders is related to hypoperfusion in particular brain regions.

\section{Materials and methods}

93 subjects referred for memory trouble participated in the study. Subjects had either Subjective (non-objective) Cognitive Impairment, Mild (Objective) Cognitive) Impairment, or Alzheimers dementia. Hypoperfusion quotes from 38 brain regions were obtained from SPECT, plus temporally resolved verb and noun fluency scores. Factor analysis was used to assess hypoperfusion in larger anatomical regions. Regression analysis was applied to the hypoperfusion factor scores and word fluency data.

\section{Results}

Impaired verb fluency was predicted by a temporal lobe hypoperfusion factor and years of education, whereas impaired noun fluency was predicted by age and hypoperfusion in the parietooccipital-parietotemporal region. Hypoperfusion in anteromedial temporal areas were more strongly associated with decreased verb fluency.

\section{Discussion}

Impaired verb fluency was predicted by a temporal lobe hypoperfusion factor and years of education, whereas impaired noun fluency was predicted by age and hypoperfusion in the parietooccipital-parietotemporal region. Hypoperfusion in anteromedial temporal areas were more strongly associated with decreased verb fluency.

\section{References}

I. Braah H, Braak E: Neuropathological stageing of Alzheimerrelated changes. Acta Neuropathologica I991, 82:239-259.

2. Piatt A, Fields JA, Paolo AM, Tröster Al: Action (verb naming) fluency as an executive function measure: convergent and divergent evidence of validity. Neuropsychologia 1999, 37:1499-1503.
3. Damasio A, Tranel D: Nouns and verbs are retrieved with differently distributed neural systems. Proceedings of the National Academy of Sciences of the United States of America 1993, 90:4957-4960.

4. Pulvermüller F: The Neuroscience of Language Cambridge: Cambridge University Press; 2003. 